\title{
Precision medicine and personalized breast cancer: combination pertuzumab therapy
}

\section{Kerry Reynolds \\ Sasmit Sarangi \\ Aditya Bardia \\ Don S Dizon}

Massachusetts General Hospital Cancer Center, Harvard Medical School, Boston, MA, USA

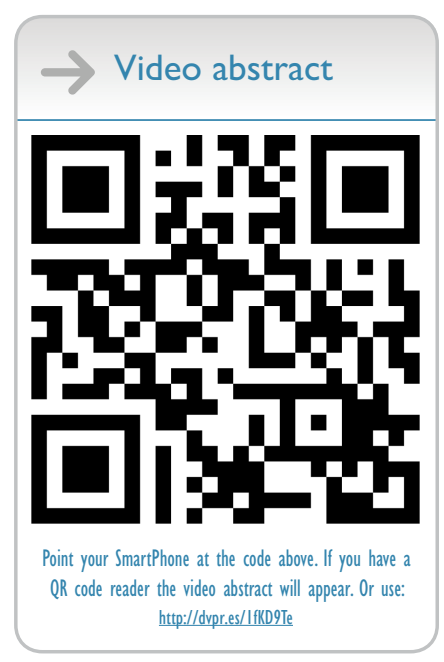

Correspondence: Don S Dizon Massachusetts General Hospital Cancer Center, Harvard Medical School, 55 Fruit Street, Boston, MA 02II4, USA Tel + I 6177244800 Email ddizon@partners.org
This article was published in the following Dove Press journal:

Pharmacogenomics and Personalized Medicine

20 March 2014

Number of times this article has been viewed

Abstract: Trastuzumab (Herceptin), a monoclonal antibody directed against the human epidermal growth-factor receptor 2 (HER2), is the poster child for antibody-based targeted therapy in breast cancer. Pertuzumab, another humanized monoclonal antibody, binds to a different domain of HER2 and prevents the formation of HER2:HER3 dimers, which is the most potent heterodimer in the HER family. The combination of trastuzumab and pertuzumab has synergistic activity, and is associated with improved clinical outcomes. The US Food and Drug Administration (FDA) approved pertuzumab in combination with trastuzumab-based chemotherapy originally as first-line therapy for metastatic HER2-positive breast cancer in 2012, and more recently as neoadjuvant therapy for localized disease in 2013. Pertuzumab is the first neoadjuvant drug to receive accelerated approval by the FDA based on pathological complete response as the primary end point. In this article, we review the mechanism of action, pharmacokinetics, clinical efficacy, safety, and current role of pertuzumab in the management of breast cancer, as well as ongoing clinical trials and future directions regarding the utility of pertuzumab as a personalized therapeutic option for HER2-positive breast cancer. In the coming years, we anticipate increased utilization of neoadjuvant trials for drug development, biomarker discovery, and validation, and envision conduct of personalized breast cancer clinics in which therapies will be routinely selected based on genetic alterations in the tumor. Regardless of the targeted therapy combinations employed based on tumor genomic profile, trastuzumab and pertuzumab will likely continue to form the backbone of the personalized regimen for HER2positive breast cancer.

Keywords: pertuzumab, HER2 breast cancer, personalized therapy, precision medicine

\section{Introduction}

Breast cancer is the most common noncutaneous cancer in the US, accounting for nearly one in three cancers diagnosed in females. It is estimated that 232,340 women in the US will get diagnosed with breast cancer in 2013. ${ }^{1}$ Human epidermal growthfactor receptor 2 (HER2) is amplified in approximately one-quarter of breast cancers, and HER2-positive tumors represent an aggressive subtype of breast cancer. ${ }^{2}$

HER2 was discovered in the mid-1980s as a transmembrane tyrosine-kinase receptor with a primary sequence very similar to that of the human epidermal growthfactor 1, and hence was termed HER2.,4 Its location on chromosomal 17 and the $185 \mathrm{kD}$ oncogene product of HER2 was coincident with the mouse neu oncogene, suggesting that the two genes were identical, and thus the term HER2-neu was coined., Subsequently, it was reported that HER2 gene amplification occurs in $20 \%-30 \%$ of human breast cancers and HER2 overexpression was a significant and independent 
predictor of time to relapse and survival among patients with breast cancer. ${ }^{6-9}$

HER2 is a member of the epidermal growth-factor receptor family, all members of which have four distinct domains in their molecular structure: an extracellular ligand-binding site, a transmembrane region, an intracellular kinase, and an intracellular C-terminal tail used for downstream signaling. In order to initiate this signaling, a ligand must bind to a receptor (HER1, HER3, or HER4), and secondary dimerization must occur. Once two family members dimerize, the kinase domain of the first receptor becomes active and phosphorylates the C-terminal tail of the other, which can then recruit scaffold and adaptor proteins for further downstream signaling. ${ }^{10}$ The HER2 receptor is the preferred dimerization partner for the other family members, and is unique in that it lacks a known ligand, but exists in a conformation that is always available to heterodimerize with other family members (HER1, HER3, and HER4).

When overexpressed, HER2 can homodimerize and initiate ligand-independent constitutive signaling. ${ }^{11}$ HER3 receptors lack a kinase domain of their own, and thus are incapable of forming homodimers. However, HER3 can form heterodimers with other HER members, and its C-terminal tail contains phosphotyrosine sites that bind with key protein domains. ${ }^{12}$ HER3 can lead to activation of multiple downstream signaling pathways, making HER2:HER3 heterodimers extremely potent signaling initiators. ${ }^{12,13}$ The formation of dimers initiates downstream signaling to several pathways, particularly the phosphoinositide 3-kinase (PI3K) and the mitogen-activated protein kinase pathways. ${ }^{13}$ Other members of the growth-factor family, such as fibroblast growth-factor receptor and insulin growth-factor receptor, can also induce intracellular signaling. ${ }^{14}$ The net consequence of these signaling events is an increase in cellular survival, proliferation, invasiveness, and angiogenesis, as outlined in Figure 1. ${ }^{11}$

While HER2-positive tumors represent an aggressive subtype of breast cancer, the prognosis of HER2-positive breast cancer has changed dramatically since the introduction of trastuzumab (Herceptin ${ }^{\circledR}$ ), a monoclonal antibody against HER2. ${ }^{15}$ Multiple large randomized clinical trials have demonstrated that trastuzumab-based chemotherapy is associated with significant improvement in response rate, time to disease progression, and overall survival in metastatic

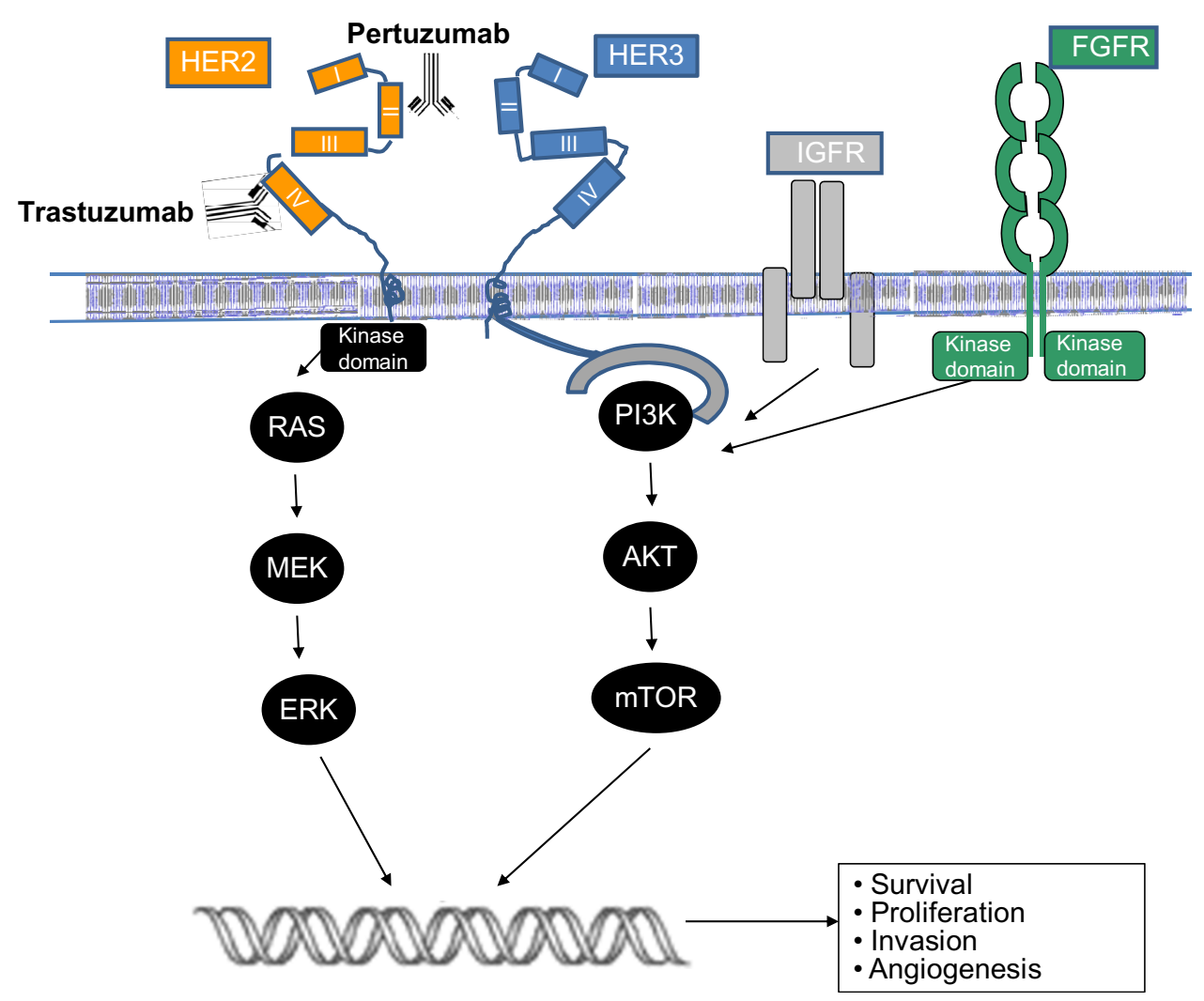

Figure I Schema outlining the activation of the human epidermal growth-factor receptor 2 pathway and antibody blockade by trastuzumab and pertuzumab. Abbreviations: HER, human epidermal growth-factor receptor; FGFR, fibroblast growth-factor receptor; IGFR, insulin-like growth-factor receptor; IV, intravenous; $\mathrm{PI3K}$, phosphoinositide 3-kinase; MEK, mitogen-activated protein kinase kinase; ERK, extracellular signal-regulated kinase; mTOR, mammalian target of rapamycin. 
breast cancer, as well as improved disease-free survival and overall survival in localized breast cancer. ${ }^{16-19}$

Despite the effectiveness of trastuzumab, a number of patients develop primary and secondary resistance. The mechanisms of trastuzumab resistance include incomplete HER2 blockade from trastuzumab alone, heterodimeric signaling from other growth factors, alteration in downstream signaling due to oncogene mutations, and loss of HER2 extracellular domain..$^{20,21}$ Several therapeutic strategies to combat this issue have emerged. A common approach is to initiate dual HER2 blockade by combining two different drugs against HER2, such as pertuzumab (Perjeta ${ }^{\circledR}$, Genentech, South San Francisco, CA, USA) and lapatinib (Tykerb ${ }^{\circledR}$, Genentech). Trastuzumab emtansine, an antibody drug conjugate, is another drug that has demonstrated efficacy in trastuzumab-resistant breast cancer. ${ }^{13,22}$ The clinical success of these therapies highlights the fact that HER2 tumors that have become resistant to trastuzumab continue to rely on HER2 signaling, and rational targeting of the pathway can overcome trastuzumab resistance.

In this review, we focus on pertuzumab and examine its 1) mechanism of action and rationale for development, 2) clinical efficacy in various settings, 3) pharmacokinetics, 4) dose and regimen, 5) safety and tolerability, and 6) current role in the management of breast cancer. We also review ongoing clinical trials and future directions regarding the utility of pertuzumab as a personalized therapeutic option for HER2positive breast cancer.

\section{Pertuzumab}

\section{Mechanism of action and rationale for development}

As demonstrated in Figure 1, trastuzumab and pertuzumab bind to different epitopes of HER2. Pertuzumab is a humanized monoclonal antibody that binds to HER2 at subdomain II, which is the extracellular epitope of the receptor involved in the dimerization of HER $2 .{ }^{23}$ Pertuzumab prevents HER2:HER3 dimer formation and subsequent HER3-mediated signaling, whereas trastuzumab binds to subdomain IV of the HER2 extracellular domain, preventing HER 2 cleavage. ${ }^{24}$ The theoretically complementary mechanisms of pertuzumab and trastuzumab imply a greater efficacy when used together, as they would provide a more complete block of downstream signaling. Both of the antibodies share common mechanisms of action, such as antibodydependent cellular cytotoxicity.

In preclinical models, the combination of pertuzumab with trastuzumab was found to reduce intracellular signaling and proliferation in breast cancer cell lines. ${ }^{25,26}$ In addition, the combination induced greater responses in tumor xenografts than either antibody alone. ${ }^{25}$ Pertuzumab has been also shown to have inhibitory effects in other tumor models, including prostate, ovarian, and lung cancer. ${ }^{27-29}$ The in vitro effects for both combination and monotherapies set the stage for evaluation of pertuzumab in clinical trials. We focus on breast cancer trials in this review.

\section{Clinical efficacy}

\section{Metastatic breast cancer}

Pertuzumab was initially explored in combination with trastuzumab as treatment for HER2-positive metastatic breast cancer in patients who had progressed on trastuzumab therapy. In a Phase II clinical trial that included advanced HER2-positive breast cancer patients with up to three lines of prior cytotoxic therapies, or those who had progressed during trastuzumab-based therapy as last treatment $(n=66)$, the pertuzumab-and-trastuzumab combination induced a response rate of $24.2 \%$, with a median progression-free survival of 5.5 months. ${ }^{30}$ The combination was well tolerated, and adverse events were minimal.

Subsequently, pertuzumab was examined as part of a first-line therapeutic combination in the pivotal CLEOPATRA (Clinical Evaluation of Pertuzumab and Trastuzumab) trial, a randomized, double-blind, placebo-controlled study consisting of 808 patients with HER2-positive metastatic breast cancer who were assigned to trastuzumab and docetaxel with either placebo or pertuzumab. ${ }^{31,32}$ The pertuzumab-based combination was associated with a significant increase in median progression-free survival from 12.4 to 18.5 months, and follow-up analysis indicated a significant increase in overall survival, with no significant difference in cardiac toxicity. ${ }^{32,33}$ Subgroup analysis of CLEOPATRA indicated that the magnitude of efficacy of dual HER2 blockade with pertuzumab and trastuzumab was comparable, irrespective of whether or not patients were trastuzumab-naïve. ${ }^{34}$ Additionally, analysis of biomarkers for prognosis or predictive value indicated that PIK3CA mutation and HER2/HER3 levels were significantly associated with a poor prognosis in both arms. ${ }^{35} \mathrm{~A}$ significant limitation of this study was the limited number of patients who had prior exposure to trastuzumab (about 11\%). This was primarily due to a lack of use of trastuzumab in the neoadjuvant or adjuvant setting at the time of patient enrollment. It is expected that ongoing trials, in particular the PHEREXA (A Study of a Combination of Trastuzumab and Capecitabine with or Without Pertuzumab in Patients with HER2-Positive Metastatic Breast Cancer) trial (Table 1), will bring greater clarity to the magnitude of benefit in the second-line setting. 
Table I Key randomized clinical trials evaluating pertuzumab therapy for HER2-positive breast cancer

\begin{tabular}{|c|c|c|c|c|}
\hline Study & Setting & Regimen & Sample size & Efficacy \\
\hline NeoSphere & Neoadjuvant & $\begin{array}{l}\text { A. Trastuzumab with docetaxel q3 weeks } * 4 \text { cycles } \\
\text { B. Pertuzumab with docetaxel q3 weeks } * 4 \text { cycles } \\
\text { C. Trastuzumab and pertuzumab with docetaxel } \\
\text { q3 weeks } * 4 \text { cycles } \\
\text { Trastuzumab and pertuzumab q } 3 \text { weeks } * 4 \text { cycles }\end{array}$ & 417 & $\begin{array}{l}P C R \\
29 \% \text { in arm } A \\
\text { vs } 24 \% \text { in arm B } \\
\text { vs } 45.8 \% \text { in arm } C \\
\text { vs } 16.8 \% \text { in arm } D \\
P=0.01 \text { C vs } A \\
P=0.03 \text { C vs } B \\
P=0.01 \text { D v } A\end{array}$ \\
\hline TRYPHAENA & Neoadjuvant & $\begin{array}{l}\text { A. Trastuzumab, pertuzumab and 5-fluorouracil, } \\
\text { epirubicin, cyclophosphamide (FEC) q3 weeks } \\
* 3 \text { cycles, followed by trastuzumab, pertuzumab, } \\
\text { and docetaxel q3 weeks *3 cycles } \\
\text { B. } 5 \text {-fluorouracil, epirubicin, and cyclophosphamide } \\
\text { (FEC) q3 weeks } * 3 \text { cycles, followed by trastuzumab, } \\
\text { pertuzumab, and docetaxel q3 weeks } * 3 \text { cycles } \\
\text { C. Trastuzumab, pertuzumab, docetaxel, and } \\
\text { carboplatin chemotherapy q3 weeks } 6 \text { cycles }\end{array}$ & 225 & $\begin{array}{l}\text { PCR in arm A: } 56.2 \% \\
\text { In arm B: } 54.7 \% \\
\text { In arm C: } 63.6 \% \\
\text { (study not powered to } \\
\text { compare the arms) }\end{array}$ \\
\hline APHINITY & Adjuvant & $\begin{array}{l}\text { Chemotherapy with trastuzumab, with or without } \\
\text { pertuzumab }\end{array}$ & 4,800 (planned) & $\begin{array}{l}\text { Trial ongoing } \\
(\mathrm{NCTO} 358877)^{66}\end{array}$ \\
\hline CLEOPATRA & $\begin{array}{l}\text { Metastatic } \\
\text { (first-line) }\end{array}$ & $\begin{array}{l}\text { Docetaxel and trastuzumab, with or without } \\
\text { pertuzumab }\end{array}$ & 808 & $\begin{array}{l}\text { Median DFS: I } 8.5 \text { vs } \\
\text { I } 2.4 \text { months }(P<0.05) \\
\text { HR } 0.62,95 \% \mathrm{Cl} 0.5 \mathrm{I}-0.75 \\
P<0.00 \text { I } \\
\text { Median OS: } 37.6 \text { months in } \\
\text { placebo arm, median OS not } \\
\text { reached in pertuzumab arm } \\
\text { HR } 0.66,95 \% \mathrm{Cl} 0.52-0.84 ; \\
P=0.0008)\end{array}$ \\
\hline MARIANNE & $\begin{array}{l}\text { Metastatic } \\
\text { (first-line) }\end{array}$ & $\begin{array}{l}\text { A. T-DMI and placebo } \\
\text { B. T-DMI and pertuzumab } \\
\text { C. Trastuzumab and taxane }\end{array}$ & I,095 (planned) & $\begin{array}{l}\text { Trial ongoing } \\
(\text { NCTOII } 20184)^{67}\end{array}$ \\
\hline PHEREXA & $\begin{array}{l}\text { Metastatic } \\
\text { (second-line) }\end{array}$ & $\begin{array}{l}\text { Capecitabine and trastuzumab, with or without } \\
\text { pertuzumab }\end{array}$ & 450 (planned) & $\begin{array}{l}\text { Trial ongoing } \\
(\text { NCTOI026142) }\end{array}$ \\
\hline
\end{tabular}

Abbreviations: HER, human epidermal growth-factor receptor; PCR, pathological complete response; DFS, disease-free survival; OS, overall survival; HR, hazard ratio; Cl, confidence interval; vs, versus; q3 weeks, every 3 weeks; NeoSphere, Neoadjuvant Study of Pertuzumab and Herceptin in an Early Regimen Evaluation; TRYPHAENA, Neoadjuvant Pertuzumab and Trastuzumab Concurrent or Sequential with an Anthracycline-Containing or Concurrent with an Anthracycline-Free Standard Regimen: A Randomized Phase II Study; APHINITY, Adjuvant Pertuzumab and Herceptin in Initial Therapy of Breast Cancer; CLEOPATRA, Clinical Evaluation of Pertuzumab and Trastuzumab; MARIANNE, A Study of Trastuzumab Emtansine (T-DMI) Plus Pertuzumab/Pertuzumab Placebo Versus Trastuzumab [Herceptin] Plus a Taxane in Patients With Metastatic Breast Cancer; PHEREXA, A Study of a Combination of Trastuzumab and Capecitabine with or Without Pertuzumab in Patients with HER2-Positive Metastatic Breast Cancer.

Pertuzumab, like other HER2-directed therapies, has minimal efficacy in HER2-negative breast cancer. In a Phase II randomized trial among women with metastatic HER2-negative breast cancer $(n=79)$, pertuzumab was found to have limited efficacy, with only $7.7 \%$ of patients achieving either a partial response or greater than 6 months of stable disease. ${ }^{36}$ In another trial among women with trastuzumab-resistant metastatic breast cancer, patients $(n=29)$ received pertuzumab monotherapy, and at the time of progression, 17 received dual blockade with pertuzumab and trastuzumab. The dual-combination therapy arm had significantly higher progressionfree survival compared to monotherapy (17.4 weeks versus 7.1 weeks), highlighting the clinical effectiveness of dual blockade. ${ }^{37}$

\section{Neoadjuvant trials}

Neoadjuvant (preoperative) therapy refers to administration of systemic therapy before surgery. Potential advantages of neoadjuvant over adjuvant therapy include downstaging of tumors to increase the likelihood of breast-conserving therapy, early initiation of systemic therapy to prevent distant metastasis, and the ability to assess in vivo tumor response to therapy by assessing pathological complete response (pCR), a surrogate marker of survival. ${ }^{38}$ Traditionally, single-agent trastuzumab-based neoadjuvant therapy has been considered the standard of care. However, a number of clinical trials have evaluated the clinical utility of dual HER2-directed therapy, and the resulting data suggest targeting multiple mechanisms may increase efficacy. A recent meta-analysis reported that dual HER2 blockade compared to trastuzumab alone did not improve breast-conserving surgery rate (relative risk [RR] 1.03, $P=0.84)$, but significantly increased rates of $\mathrm{pCR}$ overall (RR 1.39, $P<0.00001$ ), with no increase in grade $3 / 4$ toxicity $(\mathrm{RR} 1.13, P=0.16) .{ }^{39}$ Higher $\mathrm{pCR}$ was associated with 
improved disease-free survival as well as overall survival, which is further evidence of $\mathrm{pCR}$ as a surrogate end point for survival in HER2-positive breast cancers. ${ }^{40}$

Two pertuzumab-containing neoadjuvant regimens merit particular attention. NeoSphere (Neoadjuvant Study of Pertuzumab and Herceptin in an Early Regimen Evaluation) enrolled 417 breast cancer patients with locally advanced, inflammatory, or early HER2-positive breast cancers $>2 \mathrm{~cm}$ to pertuzumab, trastuzumab, both with docetaxel, or both without docetaxel. ${ }^{41}$ The schema of the NeoSphere trial is outlined in Figure 2. Patients treated with dual HER2 blockade plus docetaxel had significantly improved $\mathrm{pCR}$ at $45.8 \%$, compared with trastuzumab plus docetaxel, with pCR of $29 \%$. Of note, those treated with dual HER2 therapy without chemotherapy had a pCR of $16.8 \%$, suggesting a subset of breast cancers might respond to dual HER2 blockade without chemotherapy. There were no significant differences in tolerability between the arms that contained chemotherapy; however, antibody-only therapy had significantly fewer serious adverse events. TRYPHAENA (Neoadjuvant Pertuzumab and Trastuzumab Concurrent or Sequential with an Anthracycline-Containing or Concurrent with an Anthracycline-Free Standard Regimen: A Randomized Phase II Study), another Phase II clinical trial, was conducted in the neoadjuvant setting to evaluate the toxicity of HER2 blockade with the chemotherapeutic agents anthracycline and carboplatin. ${ }^{42}$
The study enrolled 225 patients with locally advanced and inflammatory HER2-positive breast cancer. Pertuzumab and trastuzumab were administered, either sequentially or concurrently with an anthracycline-containing regimen or concurrently with an anthracycline-free regimen, to determine the safety profile. Regardless of the chemotherapy chosen, the combination of pertuzumab with trastuzumab in the neoadjuvant setting resulted in high pCR rates $(45.3 \%-66 \%)$. These two neoadjuvant trials led to an accelerated approval of pertuzumab by the US Food and Drug Administration (FDA) as a neoadjuvant therapy (see the "Current role in management of breast cancer" section).

\section{Adjuvant trials}

While trastuzumab has an established role in the adjuvant therapy of HER2-positive patients, the role of adjuvant pertuzumab is not clear. ${ }^{16,43}$ The ongoing APHINITY (Adjuvant Pertuzumab and Herceptin in Initial Therapy of Breast Cancer) trial will evaluate the benefit of dual blockade in adjuvant therapy with trastuzumab and chemotherapy in patients who have undergone resection for HER2-positive breast cancer. ${ }^{44}$ A schema of the APHINITY trial design is shown in Figure 3. The primary outcome is disease free survival among the targeted 3,806 participants, with secondary outcomes including overall survival, quality of life, and safety, including cardiac safety. The initial results from this trial are anticipated in 2016

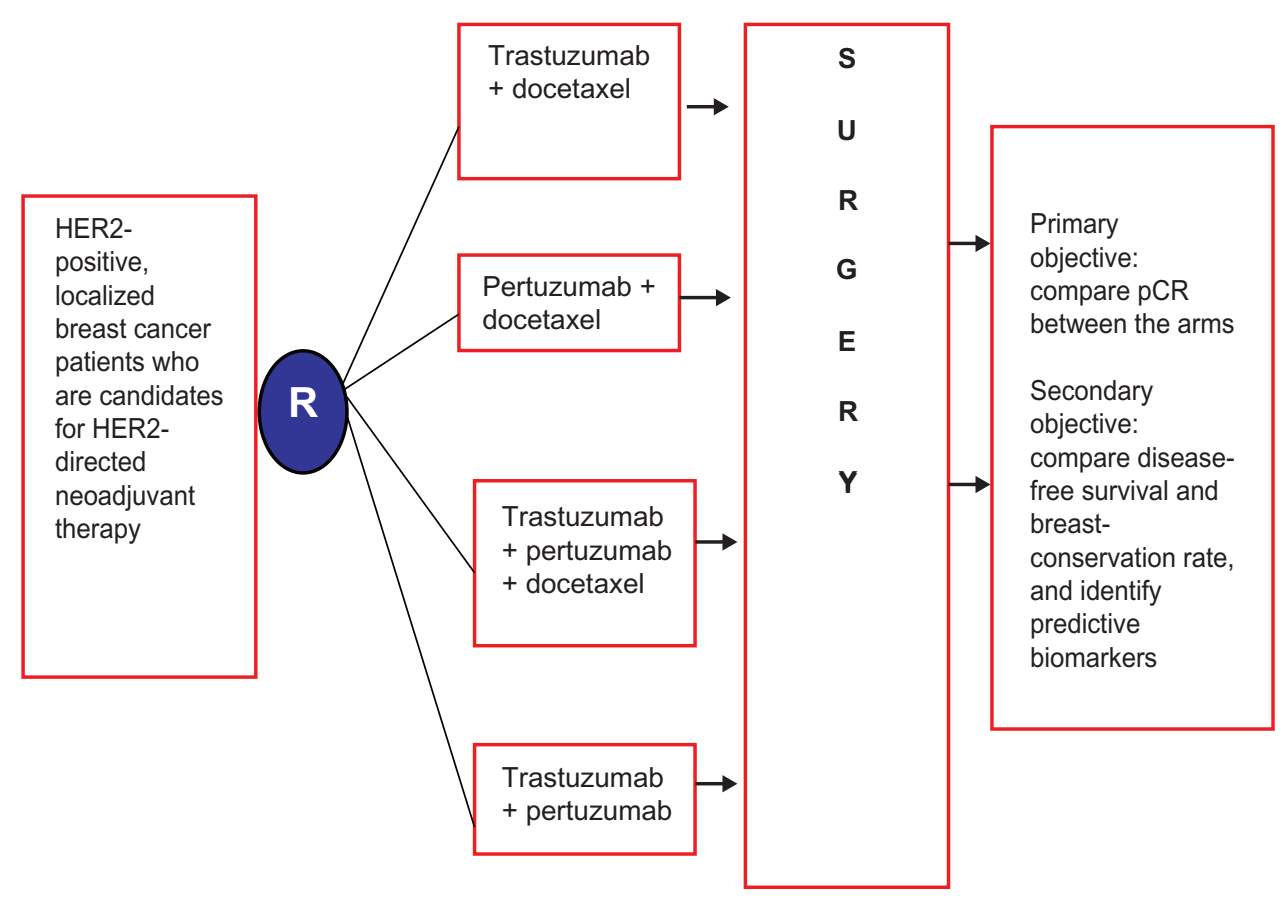

Figure 2 Schema of the NeoSphere clinical trial comparing various neoadjuvant HER2-directed therapies for localized HER2-positive breast cancer. Abbreviations: HER, human epidermal growth-factor receptor; $P C R$, pathological complete response. 


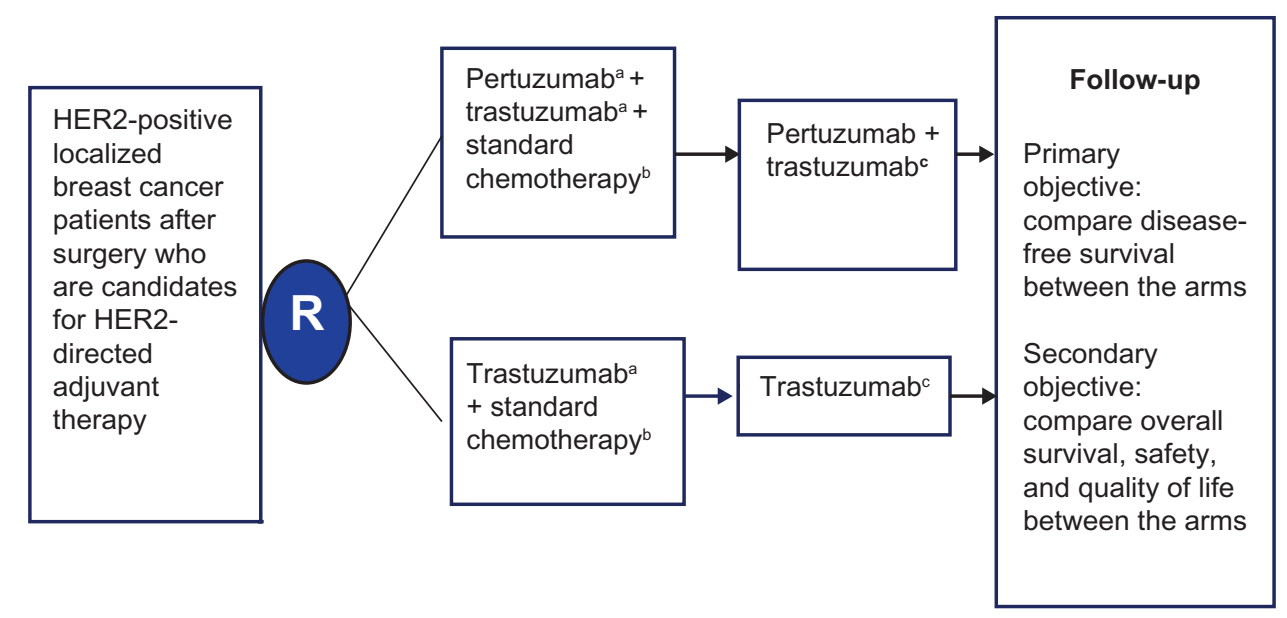

Figure 3 Schema of the APHINITY clinical trial evaluating efficacy of adjuvant pertuzumab based therapy in localized HER2-positive breast cancer.

Notes: ${ }^{a}$ Trastuzumab $6 \mathrm{mg} / \mathrm{kg}$ IV q3 weeks, pertuzumab $420 \mathrm{mg} \mathrm{IV} \mathrm{q3}$ weeks; beither anthracycline-based regimen with a taxane, or Taxotere with carboplatin; 'HER2 therapy for I year (52 weeks).

Abbreviations: HER, human epidermal growth-factor receptor; IV, intravenous; q3 weeks, every 3 weeks; APHINITY, Adjuvant Pertuzumab and Herceptin in Initial Therapy of Breast Cancer.

and will be pivotal in clarifying the role and long term safety of pertuzumab in the adjuvant setting.

\section{Review of pharmacokinetics}

Pertuzumab shows a pharmacokinetic profile similar to most immunoglobulin $\mathrm{G}$ antibodies, including trastuzumab. It has a mean half-life of about 3 weeks, and is well tolerated up to a dose of $25 \mathrm{mg} / \mathrm{kg} .{ }^{45}$ Some degree of individual variability and linear pharmacokinetics has been observed. Current dosing recommendations consist of infusing a loading dose of $840 \mathrm{mg}$ and administering $420 \mathrm{mg}$ every 3 weeks. The pharmacokinetics are affected only slightly by body weight or body surface area and are unaltered in the elderly, and thus dose adjustment is not required. ${ }^{46}$

\section{Safety and tolerability}

\section{Adverse effects}

In general, pertuzumab is safe and well tolerated; however, it is recommended to observe patients for a period of 60 minutes after the first infusion and 30 minutes after subsequent infusions. While less than $1 \%$ of the infusion reactions in the CLEOPATRA trial were grade $3-4$, if one of these serious reactions does occur, the infusion should be interrupted and appropriate rescue therapy administered. The patient should be monitored closely until they return to baseline, and providers should consider permanent discontinuation of pertuzumab therapy. For patients randomized to the pertuzumab-based regimen within the trial, mild day 1 infusion reactions occurred in up to $13.0 \%$ in the pertuzumab treated group versus $9.8 \%$ in control group. Overall, the most common ( $>30 \%)$ adverse reactions were diarrhea, alopecia, neutropenia, nausea, fatigue, rash, and peripheral neuropathy, while the most common grade 3-4 adverse reactions (2\%) were febrile neutropenia, neutropenia, leukopenia, diarrhea, peripheral neuropathy, anemia, asthenia, and fatigue ${ }^{47}$ More serious adverse reactions are rare, and include anaphylactic reactions, drop in left ventricular ejection fraction (EF), and negative effects on fetal development in utero. Adverse reactions resulted in discontinuation of therapy in $6.1 \%$ of the patients in the pertuzumab arm versus $5.3 \%$ in control arm. ${ }^{32}$

\section{Pregnancy}

Pertuzumab use is not recommended for women who are pregnant or breastfeeding. It is imperative to verify status of pregnancy prior to starting pertuzumab, and contraception is recommended during and for 6 months after therapy. Preclinical studies in animals have resulted in oligohydramnios, delayed renal development, and even fetal death. If pertuzumab is unknowingly administered during pregnancy, or if a patient becomes pregnant while receiving pertuzumab, that information should be immediately reported to the Genentech Adverse Event Line. In addition, those women should be persuaded to enroll in the MotHER Pregnancy Registry. ${ }^{48}$

\section{Immunogenicity}

In general, monoclonal antibodies have a potential for immunogenic responses in the form of antibody production. Anti-pertuzumab antibodies have been noted in a small proportion of patients; however, the pharmacokinetic impact or clinical significance of these antibodies is not known. In the CLEOPATRA trial, approximately $2.8 \%$ of patients in the 
pertuzumab group and $6.2 \%$ of patients in the placebo-treated group tested positive for anti-pertuzumab antibodies. ${ }^{32}$ The presence of pertuzumab antibodies despite no pertuzumab being administered is not well understood, though it is possible the assay is detecting antibodies to trastuzumab. The presence of antibodies did not correlate with infusion reactions.

\section{Cardiac toxicity}

Cardiac toxicity is associated with anti-HER2 therapies, particularly trastuzumab, which occurs due to a toxic effect on cardiac myocytes; however, pertuzumab appears to have lower cardiac toxicity than trastuzumab. ${ }^{49,50}$ In the CLEOPATRA study, left ventricular dysfunction was documented in $4.4 \%$ of patients in the pertuzumab-treated group versus $8.3 \%$ of patients in the placebo group. Grade 3 left ventricular systolic dysfunction occurred in $1.2 \%$ of patients in the pertuzumab treated group versus $2.8 \%$ of patients in the placebo group. ${ }^{32}$ It is thought that patients with prior anthracycline use or chest radiation may be at higher risk for decline of EF; therefore, it is recommended that patients should have echocardiography at baseline and every 3 months while on pertuzumab therapy. If a patient experiences a drop in $\mathrm{EF}$ to $<40 \%$, or EF of $40 \%-45 \%$ with a $10 \%$ absolute decrease from baseline, the provider should hold pertuzumab (and trastuzumab) for at least 3 weeks. Therapy can be reinitiated if the left ventricular EF on repeat echocardiography at 3 weeks recovers to $>45 \%$, or $40 \%-45 \%$ if associated with a less than $10 \%$ absolute decrease from baseline. If recovery is not demonstrated, discontinuation of therapy should be considered. It is also important to note that clinical trials to date have not included patients with a baseline $\mathrm{EF}$ of $<50 \%$, history of congestive heart failure, drop in EF to $<50 \%$ on trastuzumab, or conditions that impair LV function (hypertension, myocardial infarction, arrhythmia), and thus the safety of pertuzumab-based therapy for such patients is not known.

\section{Current role in management of breast cancer}

Pertuzumab (Perjeta) was FDA-approved in 2012, with a primary indication of first-line use in metastatic breast cancer in combination with trastuzumab and docetaxel. This approval was based primarily on results from the CLEOPATRA study. In September 2013, based on the results from neoadjuvant trials (Table 1), the FDA granted accelerated approval to neoadjuvant pertuzumab in combination with trastuzumab-based chemotherapy for patients with localized, locally advanced, inflammatory, or early ( $>2 \mathrm{~cm}$ tumor size or lymph nodepositive) HER2-positive breast cancer. ${ }^{51}$

The pertuzumab-based regimens, as per the current FDA prescribing label, are listed in Table $2 .{ }^{52}$ The recommended loading dose of pertuzumab is $840 \mathrm{mg}$ given as an intravenous infusion over 60 minutes. Subsequent doses are $420 \mathrm{mg}$ given

Table 2 Pertuzumab-based chemotherapy regimens as per the US Food and Drug Administration prescribing label (updated September 2013)

\begin{tabular}{|c|c|}
\hline Regimen & Dose and frequency \\
\hline \multicolumn{2}{|c|}{ Neoadjuvant regimen } \\
\hline FEC-THP* & $\begin{array}{l}\text { 5-Flurouracil } 600 \mathrm{mg} / \mathrm{m}^{2} \text {, concurrent with } \\
\text { epirubicin } 90 \mathrm{mg} / \mathrm{m}^{2} \text {, concurrent with } \\
\text { cyclophosphamide } 600 \mathrm{mg} / \mathrm{m}^{2} \text {, concurrent with } \\
\text { every } 3 \text { weeks } * 3 \mathrm{cycles} \text {, followed by Docetaxel } \\
75 \mathrm{mg} / \mathrm{m}^{2} \text {, concurrent with } \\
\text { pertuzumab loading dose of } 840 \mathrm{mg} \text {, followed by } \\
420 \mathrm{mg} \text {, concurrent with } \\
\text { trastuzumab } 8 \mathrm{mg} / \mathrm{kg} \text { loading dose, and then } 6 \mathrm{mg} / \mathrm{kg} \\
\text { every } 3 \text { weeks } * 4 \mathrm{cycles} \text {, followed by } \\
\text { trastuzumab alone } 6 \mathrm{mg} / \mathrm{kg} \text { every } 3 \text { weeks, to } \\
\text { complete a total of } 52 \text { weeks of treatment }\end{array}$ \\
\hline FECHP-THP* & $\begin{array}{l}\text { 5-Flurouracil } 600 \mathrm{mg} / \mathrm{m}^{2} \text {, concurrent with } \\
\text { epirubicin } 90 \mathrm{mg} / \mathrm{m}^{2} \text {, concurrent with } \\
\text { cyclophosphamide } 600 \mathrm{mg} / \mathrm{m}^{2} \text {, concurrent with } \\
\text { pertuzumab loading dose of } 840 \mathrm{mg} \text {, followed by } \\
420 \mathrm{mg} \text {, concurrent with } \\
\text { trastuzumab } 8 \mathrm{mg} / \mathrm{kg} \text { loading dose, and then } 6 \mathrm{mg} / \mathrm{kg} \\
\text { every three weeks } 3 \text { cycles, followed by Docetaxel } \\
75 \mathrm{mg} / \mathrm{m}^{2} \text {, concurrent with } \\
\text { pertuzumab loading dose of } 840 \mathrm{mg} \text {, followed by } \\
420 \mathrm{mg} \text {, concurrent with } \\
\text { trastuzumab } 8 \mathrm{mg} / \mathrm{kg} \text { loading dose, and then } 6 \mathrm{mg} / \mathrm{kg} \\
\text { every } 3 \text { weeks } * 4 \mathrm{cycles} \text {, followed by } \\
\text { trastuzumab alone } 6 \mathrm{mg} / \mathrm{kg} \text { every } 3 \text { weeks, to } \\
\text { complete a total of } 52 \text { weeks of treatment }\end{array}$ \\
\hline P-TCH & $\begin{array}{l}\text { Pertuzumab loading dose of } 840 \mathrm{mg} \text {, followed by } \\
420 \mathrm{mg} \text {, concurrent with } \\
\text { trastuzumab } 8 \mathrm{mg} / \mathrm{kg} \text { loading dose, and then } 6 \mathrm{mg} / \mathrm{kg} \text {, } \\
\text { concurrent with } \\
\text { docetaxel } 75 \mathrm{mg} / \mathrm{m}^{2} \text {, concurrent with } \\
\text { carboplatin AUC } 6 \\
\text { every three weeks } * 6 \text { cycles, followed by } \\
\text { trastuzumab alone } 2 \mathrm{mg} / \mathrm{kg} \text { weekly or } 6 \mathrm{mg} / \mathrm{kg} \text { every } \\
3 \text { weeks, to complete a total of } 52 \text { weeks of } \\
\text { treatment }\end{array}$ \\
\hline Metastatic regi & nen \\
\hline $\begin{array}{l}\text { Pertuzumab and } \\
\text { trastuzumab }\end{array}$ & $\begin{array}{l}\text { Trastuzumab } 8 \mathrm{mg} / \mathrm{kg} \text { loading dose, followed by } \\
6 \mathrm{mg} / \mathrm{kg} \text {, concurrent with } \\
\text { pertuzumab loading dose of } 840 \mathrm{mg} \text {, followed by } \\
420 \mathrm{mg} \text {, concurrent with } \\
\text { docetaxel } 75-100 \mathrm{mg} / \mathrm{m}^{2} \text { (at least } 6 \text { cycles) } \\
\text { every } 3 \text { weeks }\end{array}$ \\
\hline
\end{tabular}

Notes: *In the US, an Adriamycin-cyclophosphamide (AC) regimen is frequently used instead of an FEC regimen. However, there are no safety or efficacy data regarding the combination of an $\mathrm{AC}$ regimen with pertuzumab.

Abbreviation: AUC, area under curve. 
as an intravenous infusion over 30-60 minutes, and there are no recommended dose reductions. ${ }^{47}$ It is important to note that the safety of neoadjuvant pertuzumab for more than six cycles in early breast cancer, or as part of a doxorubicin-containing regimen, has not been established. Also, cost issues need to be balanced against potential benefits. ${ }^{53}$

Pertuzumab is the first neoadjuvant drug to receive accelerated approved by the FDA based on pCR as the primary end point. The full approval is contingent on the final results of APHINITY, the confirmatory study detailed in both Figure 3 and in the "Neoadjuvant trials" section of this review. The FDA reserves the right to withdraw approval for pertuzumab in the neoadjuvant setting if the results of APHINITY are negative. This accelerated process represents a paradigm shift in FDA approval, and may replace the longstanding model of drug development in oncology, which is protracted, inefficient, and costly. The neoadjuvant trial model offers a platform for rapid and efficient triage of drugs, as well as biomarker discovery to facilitate development of personalized therapy for breast cancer, and is reviewed in the next section.

\section{Future directions}

In the current era of targeted and personalized therapeutics in cancer, the classic approach of "one size fits all" cancer therapy is increasingly becoming obsolete. Since the remarkable successes of trastuzumab for HER2 breast cancer, there have been great strides in the development of targeted therapies. The field of breast cancer, particularly HER2-positive breast cancer, is now expanding with the addition of multiple active targeted therapies. In the future, we anticipate the routine use of genomics to identify biomarkers and accordingly select patients who will optimally benefit from particular anti-HER2 agents or therapy combinations. In addition, we expect increased utilization of neoadjuvant trials for drug development, biomarker discovery, and validation. These issues are discussed in the following sections.

\section{Role of biomarkers and genome sequencing}

Genome sequencing of tumor specimens is gaining increased traction in routine clinical practice. ${ }^{54}$ We anticipate that over the coming years, next-generation sequencing will be utilized routinely for discovery of genomic alterations in tumors. While promising, it should be noted that routine tumor sequencing does pose a number of challenges. Besides the ethical and regulatory issues, tumor sequencing generates an enormous amount of data, with the consequent challenge of unscrambling "driver" mutations from "passenger" mutations. As we move in the genomic era, it will be particularly important to focus on driver mutations that are "actionable", ie, modulated by a targeted therapy, and design biomarkerstratified clinical trials appropriately. ${ }^{55}$

For HER2-positive breast cancer, it will be particularly important to identify oncogene mutations in the PI3K pathway, not only because PI3K mutations predict poor therapeutic response to trastuzumab and pertuzumab but also that tumors harboring a PI3K mutation can be subject to inhibition by PI3K inhibitors. ${ }^{56,57}$ Similarly, overexpression of HER3 is a biomarker that is associated with poor prognosis and lower response to HER2 therapies, including pertuzumab, and combination with anti-HER3 therapies would be potentially important for this subgroup. ${ }^{58-60}$ Obviously, these biomarker-driven hypotheses will need to be investigated and validated before they can be incorporated into clinical practice. We anticipate that neoadjuvant trials will increasingly be utilized for this purpose, as discussed in the next section.

\section{Role of neoadjuvant trials}

Neoadjuvant trials utilizing $\mathrm{pCR}$, a validated surrogate end point for survival, as the primary end point provide an attractive platform for rapid triage of drug efficacy, biomarker identification, and validation. ${ }^{61}$ The FDA approval of neoadjuvant pertuzumab based on $\mathrm{pCR}$ as the primary end point serves as a validation of this drug-development model. ${ }^{62}$ Over the coming years, there will be a marked increase in the utilization of neoadjuvant trials in breast clinics, not only for drug approval of novel agents but also to compare different combinations for synergistic HER2 blockade, to identify predictive biomarkers for individual therapies, and to determine the optimal sequencing of HER2-blocking agents. ${ }^{63}$ Neoadjuvant trials testing combination therapies that have the potential of overcoming treatment resistance by inhibiting compensatory cross talk between pathways, such as the combination of HER2 directed therapies with PI3K inhibitors, or anti-HER3 therapies, will be particularly valuable. Similarly, for HER2positive tumors that are also hormone receptor-positive, it would be important to incorporate therapies that also target the estrogen receptor, since the combination of neoadjuvant endocrine and HER2 therapy can have additive therapeutic efficacy ${ }^{64}$ Safety is a key issue for neoadjuvant trials, and it will be important to consider carefully the amount of safety data present, avoid drugs with significant overlapping toxicities, and utilize innovative protocol development like the adaptive study design. ${ }^{65}$ 


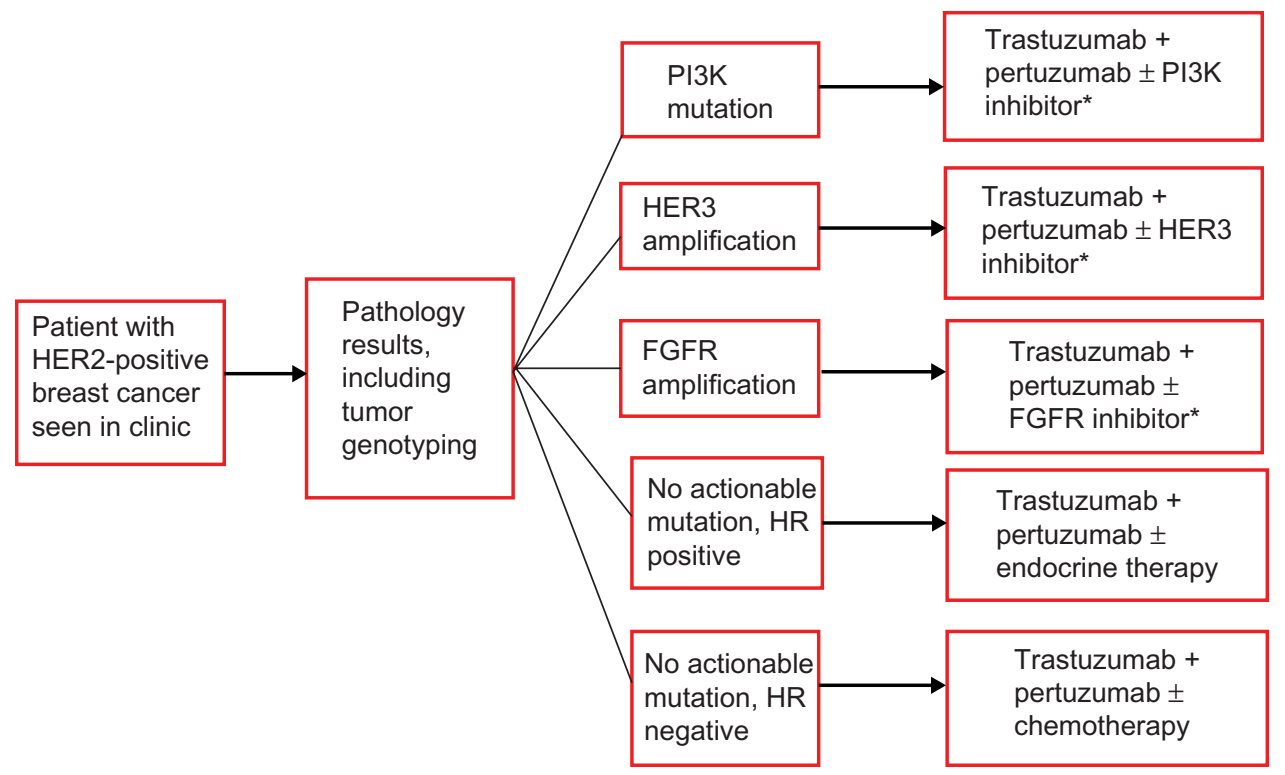

Figure 4 Schema of personalized therapy selection based on molecular profiling of breast cancer.

Notes: The purpose of this figure to provide a conceptual framework of how trastuzumab and pertuzumab would form the backbone of personalized HER2-directed therapy. It is not meant to be inclusive of all possible therapies or combinations. The various inhibitors are being investigated in clinical trials, and are not currently the standard of care. * \pm Chemotherapy, and endocrine therapy if HR positive.

Abbreviations: PI3K, phosphoinositide 3-kinase; HER, human epidermal growth-factor receptor; FGFR, fibroblast growth-factor receptor; HR, hormone receptor.

In the coming years, we envision personalized breast cancer clinics in which therapies will be routinely selected based on genetic alterations in tumors. A potential example of such a personalized therapy selection for HER2-positive breast cancer is outlined in Figure 4. Trastuzumab and pertuzumab will likely form the backbone of the various targeted therapy combinations employed in the personalized regimen.

\section{Disclosure}

The authors report no conflicts of interest in this work.

\section{References}

1. American Cancer Society. Cancer Facts and Figures: 2013-2014. Atlanta: American Cancer Society; 2013.

2. Slamon DJ, Clark GM, Wong SG, Levin WJ, Ullrich A, McGuire WL. Human breast cancer: correlation of relapse and survival with amplification of the HER-2/neu oncogene. Science. 1987;235(4785):177-182.

3. Coussens L, Yang-Feng TL, Liao YC, et al. Tyrosine kinase receptor with extensive homology to EGF receptor shares chromosomal location with neu oncogene. Science. 1985;230(4730):1132-1139.

4. King CR, Kraus MH, Aaronson SA. Amplification of a novel v-erbB-related gene in a human mammary carcinoma. Science. 1985;229(4717):974-976.

5. Schechter AL, Stern DF, Vaidyanathan L, et al. The neu oncogene: an erb-B-related gene encoding a $185,000-\mathrm{Mr}$ tumour antigen. Nature. 1984;312(5994):513-516.

6. Tandon AK, Clark GM, Chamness GC, Ullrich A, McGuire WL. HER-2/ neu oncogene protein and prognosis in breast cancer. J Clin Oncol. 1989;7(8):1120-1128.

7. Toikkanen S, Helin H, Isola J, Joensuu H. Prognostic significance of HER-2 oncoprotein expression in breast cancer: a 30-year follow-up. J Clin Oncol. 1992;10(7):1044-1048.
8. Seshadri R, Firgaira FA, Horsfall DJ, McCaul K, Setlur V, Kitchen P. Clinical significance of HER-2/neu oncogene amplification in primary breast cancer. The South Australian Breast Cancer Study Group. J Clin Oncol. 1993;11(10):1936-1942.

9. Marks JR, Humphrey PA, Wu K, et al. Overexpression of p53 and HER-2/neu proteins as prognostic markers in early stage breast cancer. Ann Surg. 1994;219(4):332-341.

10. Pegram MD, Konecny G, Slamon D. The molecular and cellular biology of HER2/neu gene amplification/overexpression and the clinical development of herceptin (trastuzumab) therapy for breast cancer. Cancer Treat Res. 2000;103:57-75.

11. Arteaga CL, Sliwkowski MX, Osborne CK, Perez EA, Puglisi F, Gianni L. Treatment of HER2-positive breast cancer: current status and future perspectives. Nat Rev Clin Onc. 2012;9(1):16-32.

12. Amin DN, Campbell MR, Moasser MM. The role of HER3, the unpretentious member of the HER family, in cancer biology and cancer therapeutics. Semin Cell Dev Biol. 2010;21(9):944-950.

13. Baselga J, Swain SM. Novel anticancer targets: revisiting ERBB2 and discovering ERBB3. Nat Rev Cancer. 2009;9(7):463-475.

14. Lu Y, Zi X, Zhao Y, Mascarenhas D, Pollak M. Insulin-like growth factor-I receptor signaling and resistance to trastuzumab (Herceptin). J Natl Cancer Inst. 2001;93(24):1852-1857.

15. Slamon DJ, Leyland-Jones B, Shak S, et al. Use of chemotherapy plus a monoclonal antibody against HER2 for metastatic breast cancer that overexpresses HER2. N Engl J Med. 2001;344(11):783-792.

16. Piccart-Gebhart MJ, Procter M, Leyland-Jones B, et al. Trastuzumab after adjuvant chemotherapy in HER2-positive breast cancer. $N$ Engl $J$ Med. 2005;353(16):1659-1672.

17. Slamon D, Eiermann W, Robert N, et al. Adjuvant trastuzumab in HER2-positive breast cancer. $N$ Engl J Med. 2011;365(14): 1273-1283.

18. McArthur HL, Mahoney KM, Morris PG, et al. Adjuvant trastuzumab with chemotherapy is effective in women with small, node-negative, HER2-positive breast cancer. Cancer. 2011;117(24):5461-5468.

19. Rodrigues MJ, Peron J, Frénel JS, et al. Benefit of adjuvant trastuzumabbased chemotherapy in T1ab node-negative HER2-overexpressing breast carcinomas: a multicenter retrospective series. Ann Oncol. 2013;24(4):916-924. 
20. Mohd Sharial MS, Crown J, Hennessy BT. Overcoming resistance and restoring sensitivity to HER2-targeted therapies in breast cancer. Ann Oncol. 2012;23(12):3007-3016.

21. Nahta R, Esteva FJ. Herceptin: mechanisms of action and resistance. Cancer Lett. 2006;232(2):123-138.

22. Verma S, Miles D, Gianni L, et al. Trastuzumab emtansine for HER2-positive advanced breast cancer. $N$ Engl J Med. 2012;367(19): 1783-1791.

23. Franklin MC, Carey KD, Vajdos FF, Leahy DJ, de Vos AM, Sliwkowski MX. Insights into ErbB signaling from the structure of the ErbB2-pertuzumab complex. Cancer Cell. 2004;5(4):317-328.

24. Kristjansdottir K, Dizon D. HER-dimerization inhibitors: evaluating pertuzumab in women's cancers. Expert Opin Biol Ther. 2010;10(2):243-250.

25. Scheuer W, Friess T, Burtscher H, Bossenmaier B, Endl J, Hasmann M. Strongly enhanced antitumor activity of trastuzumab and pertuzumab combination treatment on HER2-positive human xenograft tumor models. Cancer Res. 2009;69(24):9330-9336.

26. Nahta R, Hung MC, Esteva FJ. The HER-2-targeting antibodies trastuzumab and pertuzumab synergistically inhibit the survival of breast cancer cells. Cancer Res. 2004;64(7):2343-2346.

27. Agus DB, Akita RW, Fox WD, et al. Targeting ligand-activated ErbB2 signaling inhibits breast and prostate tumor growth. Cancer Cell. 2002;2(2):127-137.

28. Langdon SP, Faratian D, Nagumo Y, Mullen P, Harrison DJ. Pertuzumab for the treatment of ovarian cancer. Expert Opin Biol Ther. 2010;10(7):1113-1120.

29. Herbst RS, Davies AM, Natale RB, et al. Efficacy and safety of single-agent pertuzumab, a human epidermal receptor dimerization inhibitor, in patients with non small cell lung cancer. Clin Cancer Res. 2007;13(20):6175-6181.

30. Baselga J, Gelmon KA, Verma S, et al. Phase II trial of pertuzumab and trastuzumab in patients with human epidermal growth factor receptor 2-positive metastatic breast cancer that progressed during prior trastuzumab therapy. J Clin Oncol. 2010;28(7):1138-1144.

31. Baselga J, Swain SM. CLEOPATRA: a phase III evaluation of pertuzumab and trastuzumab for HER2-positive metastatic breast cancer. Clin Breast Cancer. 2010;10(6):489-491.

32. Baselga J, Cortés J, Kim SB, et al. Pertuzumab plus trastuzumab plus docetaxel for metastatic breast cancer. $N$ Engl $J$ Med. 2012;366(2):109-119.

33. Swain SM, Kim SB, Cortés J, et al. Pertuzumab, trastuzumab, and docetaxel for HER2-positive metastatic breast cancer (CLEOPATRA study): overall survival results from a randomised, double-blind, placebo-controlled, phase 3 study. Lancet Oncol. 2013;14(6): 461-471.

34. Ciruelos Gil EM, Brufsky A, Im YH, et al. Efficacy and safety of first-line (1L) pertuzumab (P), trastuzumab (T), and docetaxel (D) in HER2-positive MBC (CLEOPATRA) in patients previously exposed to trastuzumab. J Clin Oncol. 2013;Suppl 31:600.

35. Baselga J, Cortés J, Im SA, et al. Biomarker analyses in CLEOPATRA: a phase III, placebo-controlled study of pertuzumab in HER-2positive, first-line metastatic breast cancer (MBC). Cancer Res. 2012;72(Suppl 24): S5-S11.

36. Gianni L, Lladó A, Bianchi G, et al. Open-label, phase II, multicenter, randomized study of the efficacy and safety of two dose levels of pertuzumab, a human epidermal growth factor receptor 2 dimerization inhibitor, in patients with human epidermal growth factor receptor 2-negative metastatic breast cancer. J Clin Oncol. 2010;28(7): 1131-1137.

37. Cortés J, Fumoleau P, Bianchi GV, et al. Pertuzumab monotherapy after trastuzumab-based treatment and subsequent reintroduction of trastuzumab: activity and tolerability in patients with advanced human epidermal growth factor receptor 2-positive breast cancer. J Clin Oncol. 2012;30(14):1594-1600.

38. Bardia A, Baselga J. Preoperative chemotherapy for operable breast cancer. In: Lippman M, Harris J, Monica Morrow M, Osborne K, editors. Diseases of the Breast. Philadelphia: Lippincott Williams \& Wilkins; 2013.
39. Reynolds KL, Bhatia A, Cheng X, et al. Neoadjuvant single and dual HER2 blockade among patients with localized HER2-positive breast cancer. J Clin Oncol. 2013;31 Supp1 26:147.

40. Bardia A, Greenup R, Moy B, et al. Pathological complete remission after neoadjuvant chemotherapy predicts improved survival in the various breast cancer subtypes: Systematic review and meta-analyses. Poster presented at: AACR Advances in Breast Cancer Research; October 12-15, 2011; San Francisco, CA.

41. Gianni L, Pienkowski T, Im YH, et al. Efficacy and safety of neoadjuvant pertuzumab and trastuzumab in women with locally advanced, inflammatory, or early HER2-positive breast cancer (NeoSphere): a randomised multicentre, open-label, phase 2 trial. Lancet Oncol. 2012;13(1):25-32.

42. Schneeweiss A, Chia S, Hickish T, et al. Neoadjuvant pertuzumab and trastuzumab concurrent or sequential with an anthracyclinecontaining or concurrent with an anthracycline-free standard regimen: a randomized phase II study (TRYPHAENA). Cancer Res. 2011;71(Suppl 24):S5-S6.

43. Gianni L, Dafni U, Gelber RD, et al. Treatment with trastuzumab for 1 year after adjuvant chemotherapy in patients with HER2-positive early breast cancer: a 4-year follow-up of a randomised controlled trial. Lancet Oncol. 2011;12(3):236-244.

44. von Minckwitz G, Baselga J, Bradbury I, et al. OT1-02-04: adjuvant pertuzumab and herceptin in initial therapy of breast cancer: APHINITY (BIG 4-11/BO25126/TOC4939g). Cancer Res. 2011;71(Suppl 24): OT1-02-04.

45. Agus DB, Gordon MS, Taylor C, et al. Phase I clinical study of pertuzumab, a novel HER dimerization inhibitor, in patients with advanced cancer. J Clin Oncol. 2005;23(11):2534-2543.

46. Ng CM, Lum BL, Gimenez V, Kelsey S, Allison D. Rationale for fixed dosing of pertuzumab in cancer patients based on population pharmacokinetic analysis. Pharm Res. 2006;23(6):1275-1284.

47. Genentech. Perjeta [full prescribing information]. San Francisco: Genentech; 2012.

48. MotHER Pregnancy Registry. Available from: http://www.herceptinpregnancyregistry.com. Accessed January 16, 2014.

49. Crone SA, Zhao YY, Fan L, et al. ErbB2 is essential in the prevention of dilated cardiomyopathy. Nat Med. 2002;8(5):459-465.

50. Perez EA. Cardiac toxicity of ErbB2-targeted therapies: what do we know? Clin Breast Cancer. 2008;8 Suppl 3:S114-S120.

51. US Food and Drug Administration. FDA approves Perjeta for neoadjuvant breast cancer treatment [press release]. Silver Spring, MD: FDA; September 30, 2013. Available from: http://www.fda.gov/NewsEvents/ Newsroom/PressAnnouncements/ucm 370393.htm. Accessed October 5, 2013.

52. US Food and Drug Administration. Pertuzumab [prescribing information]. Available from: http://www.accessdata.fda.gov/ drugsatfda_docs/label/2013/125409s051lbl.pdf?et_cid=32583475\&et_r $\mathrm{id}=463654371 \&$ linkid $=+\mathrm{http} \% 3 \mathrm{a} \% 2 \mathrm{f} \% 2 \mathrm{fwww}$.accessdata.fda. gov\%2fdrugsatfda_docs\%2flabel\%2f2013\%2f125409s051lbl.pdf. Accessed on October 5, 2013

53. Chernew ME, Newcomer LN, Swain SM. Treatment and cost implications of pertuzumab. Am J Manag Care. 2012;18(4): SP151-SP153.

54. Banerji S, Cibulskis K, Rangel-Escareno C, et al. Sequence analysis of mutations and translocations across breast cancer subtypes. Nature. 2012;486(7403):405-409.

55. Sleijfer S, Bogaerts J, Siu LL. Designing transformative clinical trials in the cancer genome era. J Clin Oncol. 2013;31(15): 1834-1841.

56. Esteva FJ, Guo H, Zhang S, et al. PTEN, PIK3CA, p-AKT, and p-p70S6K status: association with trastuzumab response and survival in patients with HER2-positive metastatic breast cancer. Am J Pathol. 2010;177(4):1647-1656.

57. Hanker AB, Pfefferle AD, Balko JM, et al. Mutant PIK3CA accelerates HER2-driven transgenic mammary tumors and induces resistance to combinations of anti-HER2 therapies. Proc Natl Acad Sci U S A. 2013;110(35):14372-14377. 
58. Ocana A, Vera-Badillo F, Seruga B, Templeton A, Pandiella A, Amir E. HER3 overexpression and survival in solid tumors: a meta-analysis. J Natl Cancer Inst. 2013;105(4):266-273.

59. Makhija S, Amler LC, Glenn D, et al. Clinical activity of gemcitabine plus pertuzumab in platinum-resistant ovarian cancer, fallopian tube cancer, or primary peritoneal cancer. J Clin Oncol. 2010;28(7):1215-1223.

60. Berghoff AS, Bago-Horvath Z, Preusser M, et al. Co-expression of HER3 in HER2-positive metastatic breast cancer patients is an independent predictor of impaired prognosis. Poster presented at: European Congress; September 27-October 1, 2013; Amsterdam, Netherlands.

61. Bardia A, Baselga J. Neoadjuvant therapy as a platform for drug development and approval in breast cancer. Clin Cancer Res. 2013;19(23): 6360-6370.

62. Prowell TM, Pazdur R. Pathological complete response and accelerated drug approval in early breast cancer. $N$ Engl $\mathrm{J} \mathrm{Med}$. 2012;366(26):2438-2441.

63. Untch M, von Minckwitz G. Neoadjuvant chemotherapy: early response as a guide for further treatment: clinical, radiological, and biological. J Natl Cancer Inst Monogr. 2011;2011(43):138-141.

64. Rimawi MF, Mayer IA, Forero A, et al. Multicenter phase II study of neoadjuvant lapatinib and trastuzumab with hormonal therapy and without chemotherapy in patients with human epidermal growth factor receptor 2-overexpressing breast cancer: TBCRC 006. J Clin Oncol. 2013;31(14):1726-1731.
65. DeMichele A, Berry DA, Zujewski J, et al. Developing safety criteria for introducing new agents into neoadjuvant trials. Clin Cancer Res. 2013;19(11):2817-2823.

66. Hoffmann-La Roche. A Study of Pertuzumab in Addition to Chemotherapy and Herceptin (Trastuzumab) as Adjuvant Therapy in Patients With HER2-Positive Primary Breast Cancer. Available from: http:// clinicaltrials.gov/show/NCT01358877. NLM identifier: NCT01358877. Accessed December 10, 2013.

67. Hoffmann-La Roche. A Study of Trastuzumab Emtansine (T-DM1) Plus Pertuzumab/Pertuzumab Placebo Versus Trastuzumab [Herceptin] Plus a Taxane in Patients With Metastatic Breast Cancer (MARIANNE). Available from: http://clinicaltrials.gov/show/NCT01120184. NLM identifier: NCT01120184. Accessed December 10, 2013.

68. Hoffmann-La Roche. A Study of a Combination of Trastuzumab and Capecitabine With or Without Pertuzumab in Patients With HER2-positive Metastatic Breast Cancer (PHEREXA). Available from: http://clinicaltrials.gov/show/NCT01026142. NLM identifier: NCT01026142. Accessed December 10, 2013.
Pharmacogenomics and Personalized Medicine

\section{Publish your work in this journal}

Pharmacogenomics and Personalized Medicine is an international, peerreviewed, open access journal characterizing the influence of genotype on pharmacology leading to the development of personalized treatment programs and individualized drug selection for improved safety, efficacy and sustainability. This journal is indexed on the American Chemical

\section{Dovepress}

Society's Chemical Abstracts Service (CAS). The manuscript management system is completely online and includes a very quick and fair peer-review system, which is all easy to use. Visit http://www.dovepress. $\mathrm{com} /$ testimonials.php to read real quotes from published authors.

Submit your manuscript here: http://www.dovepress.com/pharmacogenomics-and-personalized-medicine-journal 